\title{
CEMENTED VERSUS UNCEMENTED FEMORAL COMPONENTS IN THE RING HIP PROSTHESIS
}

\author{
S. P. GODSIFF, R. J. H. EMERY, M. B. HEYWOOD-WADDINGTON, T. L. THOMAS
}

From Black Notley Hospital, Braintree

\begin{abstract}
A prospective, randomised and independently assessed trial of the Ring UPM total hip replacement showed that the quality of the early result was better if the femoral prosthesis was cemented than if it was not.

More patients with cemented prostheses were painfree at four months ( $58 \%$ cemented:42\% uncemented) and at one year (63\% cemented:50\% uncemented), but at two years pain relief was equal in both groups. At two years significantly more patients with cemented prostheses could walk without support (96\% cemented:62\% uncemented, $p=0.01$ to 0.05 ).

There is a need for more similar trials to compare the results of contemporary designs of cemented and uncemented total hip prostheses.
\end{abstract}

In total hip replacement (THR), satisfactory long-term results have been reported with cemented and uncemented femoral components. Comparison between the two methods is difficult, however, because different implants and instruments have been used and few controlled prospective studies have been reported (Gross 1988). One such study suggested that the early results with uncemented femoral components were inferior to those with cemented implants, but there were considerable differences between the components used in the two groups (Wykman and Goldie 1984).

Our aim was to compare the short-term results of fixation with and without cement of the Ring UPM total hip prosthesis in a prospective, randomly controlled trial.

\section{PATIENTS AND METHODS}

Between April 1986 and September 198954 patients (58 hips) with a diagnosis of primary osteoarthritis of the hip were entered into the trial. The main criteria for inclusion

S. P. Godsiff, FRCS, Orthopaedic Registrar

M. B. Heywood-Waddington, FRCS, Consultant Orthopaedic Surgeon Broomfield Hospital, Court Road, Broomfield, Chelmsford, Essex CMI SET, England.

R. J. H. Emery, MS, FRCS Ed, Consultant Orthopaedic Surgeon Central Middlesex Hospital, Acton Lane, Park Royal, London NW10 7NS, England.

T. L. Thomas, FRCS, Consultant Orthopaedic Surgeon Black Notley Hospital, Braintree, Essex CM7 8NF, England.

Correspondence should be sent to Mr S. P. Godsiff at 7, Hancock Court, Norwich NR5 9NN, Norfolk, England.

(C) 1992 British Editorial Society of Bone and Joint Surgery

0301-620X/92/6451 \$2.00

J Bone Joint Surg [Br] 1992; 74-B:822-4. were age between 55 and 75 years, no arthritis in other joints, and no neurological or medical condition causing locomotor disability.

All patients gave their informed consent and were allocated randomly to receive either cemented or uncemented implants by choosing one of two sealed envelopes. They were not told which group they were entering.

The same design of prosthesis was used in both groups. The Ring UPM total hip prosthesis (Zimmer Ltd, Swindon, England; Fig. 1) has an acetabular component of high-density polyethylene, and a femoral component of titanium alloy with titanium mesh forgings on the proximal part of the stem for tissue ingrowth. The acetabular components were all implanted without cement.

The operations were performed by one of two surgeons (MBH-W or TLT); both used the posterior approach as described by Ring (1968). The acetabulum and femur were prepared in a similar fashion in both groups except that in the cemented group the femoral shaft was irrigated, brushed and vented before cementing. Methylmethacrylate cement with gentamicin (Palacos-R; Schering-Plough Ltd, Mildenhall, England) was inserted with a gun, and a cement restrictor was used distally to allow pressurisation.

Postoperatively, all the patients had active and assisted exercises and were allowed to walk with two crutches at seven days. Crutch walking was maintained for three months.

The femoral component was cemented in 30 hips and uncemented in 28 . There were 20 women in the cemented group and 16 in the uncemented group. The average age of the cemented group was 64.4 years ( 55 to 
74 ) and of the uncemented group 64.5 years (55 to 74). The mean pre-operative scores for pain $(4.0$ cemented: 3.7 uncemented), activity ( 3.7 cemented $: 3.2$ uncemented) and the use of walking aids ( 2.4 cemented: 2.3 uncemented) were similar in both groups.

Assessments were made at four months, one year

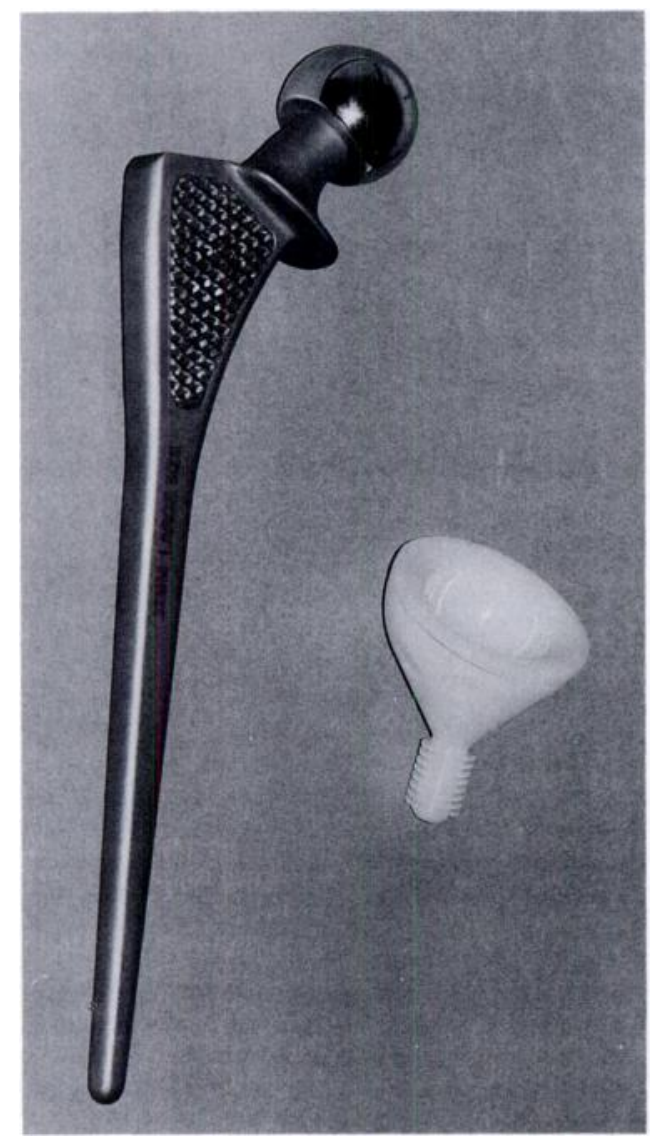

Fig. 1

The Ring UPM prosthesis.

Table I. Scales of pain, activity and use of walking aids after total hip replacement with the Ring UPM prosthesis

\begin{tabular}{ll}
\hline & Score \\
\hline Pain & \\
None & 1 \\
Slight & 2 \\
Moderate & 3 \\
Severe & 4 \\
Disabling & 5 \\
& \\
Limitation of activity & \\
None & 1 \\
Slight & 2 \\
Moderate & 3 \\
Severe & 4 \\
Gross & 5 \\
& \\
Use of walking aids & \\
None & 1 \\
One stick outdoors & 2 \\
One support at all times & 3 \\
Bilateral support & 4 \\
Frame & 5 \\
\hline
\end{tabular}

and two years by an independent, non-orthopaedic medical practitioner, who was unaware of the type of femoral fixation which had been used. A pro forma was used to record the quality of the result by reference to a five-point scale of pain, activity and need for walking aids (Table I). Radiographs were taken and studied for evidence of component loosening or subsidence.

All patients attended their one-year review; 26 cemented and 21 uncemented hips were reviewed at four months and 23 cemented and 24 uncemented hips at two years. The remainder were lost to follow-up. The chisquared test was used to compare the outcome in the two groups.

\section{RESULTS}

At four months, 15 (58\%) of the cemented group had no pain compared with only nine $(43 \%)$ of the uncemented group ( $p=0.25$ to 0.5 ). Seventeen $(65 \%)$ of the cemented group used only one stick or none compared with 12 $(57 \%)$ of the uncemented group ( $p=0.25$ to 0.5 ). Both groups had similar ranges of activity; $17(65 \%)$ cemented hips and $13(61 \%)$ uncemented hips allowed full or only slightly limited activity.

At one year, $19(63 \%)$ of the cemented group were painfree compared with $14(50 \%)$ of the uncemented group ( $p=0.25$ to 0.5$)$. Twenty-three patients $(77 \%)$ with cemented implants used no walking aids compared with $17(60 \%)$ with uncemented implants $(p=0.1$ to $0.25)$. In the cemented group, $21(70 \%)$ of patients were fully active compared with $22(79 \%)$ in the uncemented group ( $\mathrm{p}=0.25$ to 0.5$)$.

When reviewed at two years the pain scores and the activity scores were similar in the two groups, with 15 of 23 cemented and 15 of 24 uncemented hips painfree with no restriction. There was, however, a significant difference between the groups in their use of walking aids: 22 $(96 \%)$ of the cemented group, but only $15(62 \%)$ of the uncemented group used no aids ( $p=0.01$ to 0.05 ).

There were no intra-operative complications and no superficial or deep infections. One patient in the cemented group suffered a postoperative pulmonary embolus and subsequent cardiac arrest. She was successfully resuscitated, made a full recovery, and went on to have an excellent result at two years.

\section{DISCUSSION}

Our findings suggest that the early results of total hip replacement with the Ring UPM prosthesis are better if the femoral component is cemented than if it is not. The most striking differences between the two groups were the incidence of pain and the use of walking aids. At four months and at one year more patients were completely painfree in the cemented group than in the uncemented group. By two years the pain scores in the uncemented patients had improved so that there was little difference between the two groups. At two years, however, 
significantly more patients with uncemented implants used walking aids.

Reliance on walking aids may be a good indicator of the quality of the result of a total hip replacement, and it is included as a factor in several hip scoring systems (Merle d'Aubigné and Postel 1954; Harris 1969). Ring (1983) stated that failure to walk independently after THR downgraded the quality of the final result. In his series of 471 uncemented metal-on-plastic hip replacements followed up for one to three years, $95 \%$ had an excellent result. In our study the patients with uncemented femoral prostheses had good pain relief and functional activity at two years, but only $60 \%$ of them could be graded as excellent on Ring's criteria because of their continued reliance on walking aids.

The results of another independent study with the Ring UPM replacement were similar to ours. In a retrospective review of 234 hips ( $56 \%$ assessed clinically, $44 \%$ assessed by questionnaire) followed up for between two and five years, Mok and Bryant (1989) found that $61 \%$ required the use of at least one support some of the time. In that study, $38 \%$ of the operations were carried out by junior surgeons with varying levels of experience with the Ring prosthesis, whereas in our series both operating surgeons had considerable experience with this implant. In our study results similar to those reported by Ring were achieved only when the femoral component was cemented.

This report concentrates on the quality of the early results. We intend to follow these patients and to compare their rates of infection, loosening and need for revision in the long term. The only difference between the cemented and the uncemented groups which achieved statistical significance $(p<0.05)$ was the reliance on walking aids at two years, but it is possible that had we sampled more patients the trends observed in the other parameters could have become significant. Our original intention was to have larger patient groups but at three to five years we began to see an unacceptable number of femoral stem breakages in both groups, and decided that it was unethical to continue the trial. This complication has been reported elsewhere by Wilson, Nolan and Heywood-Waddington in 1992, and we no longer use this prosthesis.
The general issue of cemented versus uncemented hip prostheses remains contentious; randomised, prospective clinical trials to evaluate surgical treatments are difficult to set up and administer. Our trial suffered from the common problems of drop-out and failure to attend for some of the assessments (Cowell and Curtis 1985). In two small but well-matched groups of patients we have shown that in the short term, uncemented femoral prostheses gave less relief of pain and caused greater reliance on walking aids than did cemented implants. More modern designs of uncemented implants should, perhaps, be subjected to a similar evaluation to see whether they provide a similarly poor quality of result in the first two years after implantation.

The authors wish to acknowledge the help of Dr Honor Malone, who was responsible for the independent assessment of the patients, and Mrs Frances Pierson for administering the trial.

No benefits in any form have been received or will be received from a commercial party related directly or indirectly to the subject of this article.

\section{REFERENCES}

Cowell HR, Curtiss PH Jr. Editorial. The randomised clinical trial. J Bone Joint Surg [Am] 1985; 67-A :1151-2.

Gross M. A critique of the methodologies used in clinical studies of hipjoint arthroplasty published in the English-language orthopaedic literature. J Bone Joint Surg [Am] 1988; 70-A :1364-71.

Harris WH. Traumatic arthritis of the hip after dislocation and acetabular fractures: treatment by mold arthroplasty. J Bone Joint Surg [ Am] 1969; 51-A :737-55.

Merle d'Aubigné R, Postel M. Functional results of hip arthroplasty with acrylic prosthesis. J Bone Joint Surg [Am] 1954; 36-A: 451-75.

Mok DWH, Bryant KM. Ring uncemented plastic-on-metal hip replacements: results from an independent unit. $J R$ Soc Med $1989 ; 82: 142-4$.

Ring PA. Complete replacement arthroplasty of the hip by the Ring prosthesis. J Bone Joint Surg [Br] 1968; 50-B :720-31.

Ring PA. Ring UPM total hip arthroplasty. Clin Orthop 1983; 176: 115-23.

Wilson LF, Nolan JF, Heywood-Waddington MB. Fracture of the femoral stem of the Ring TCH hip prosthesis. J Bone Joint Surg $[\mathrm{Br}] 1992 ; 74-\mathrm{B}$ : in press.

Wykman A, Goldie I. A prospective randomised study comparing results of cemented and non-cemented endoprosthetic fixation in the surgical treatment of osteoarthrosis of the hip. Eng Med 1984; $13: 181-4$. 